\title{
The Effects of High-Molecular-Weight Components on the Viscoelastic Properties of Polystyrene
}

\author{
W. M. Prest, Jr.* and Roger S. Porter \\ Department of Polymer Science and Engineering, University of Massachusetts, \\ Amherst, Massachusetts 01002, U.S.A.
}

(Received March 11, 1972)

\begin{abstract}
The effects of molecular weight distribution (MWD) on the steady-state shear and the dynamic viscoelastic properties of high-molecular-weight $(M W>97000)$ polystyrene have been examined with a Weissenberg rheogoniometer. Synthetic MWDs were produced by blending polystyrene components of defined MWs and narrow MWDs. Experiments were designed to examine the effects of small amounts of a high-MW component on the viscous and elastic properties of the melt.

The frequency response of the storage and loss moduli, $G^{\prime}$ and $G^{\prime \prime}$, and the storage compliance $\boldsymbol{J}^{\prime}$, are compared with the shear rate dependence of the shear stress $\tau_{12}$, the primary normal stress difference $P_{11}-P_{22}$, and the shear modulus $G$, as a function of the composition of the blend. At low deformation rates the dynamic and the steadystate shear properties are equal; $G^{\prime \prime}=\tau_{12}, G^{\prime}=\left(P_{11}-P_{22}\right) / 2$, and $G=1 / J^{\prime}$. At higher deformation rates the steady-state shear properties are primarily controlled by the highMW component while the dynamic properties reflect the response of each component. The zero shear viscosities of the blends and the narrow-MWD components exhibit the same dependence on the weight-average MW. Four predictions for the MWD dependence of the steady-state shear compliance $J_{e}^{\circ}$ are compared with the experimental results. Although overestimating the magnitude in the maximum in $J_{\Theta}^{\circ}$, only Graessley's theory accounts for the observed behavior on either side of the maximum.

The data is used to discuss the previously reported dependence of the characteristic relaxation time, $\tau=\eta_{0} J_{\mathrm{e}}^{\circ}$, on the shape of the non-Newtonian flow response. The $\tau$ is found to be a measure of the time scale of the relaxation processes of the high-MW component.
\end{abstract}

KEY WORDS Blend / Molecular Weight Distribution / Viscoelasticity / Rheology / Polystyrene / non-Newtonian Flow /

The viscoelastic properties of polymer melts are strongly dependent on molecular weight (MW) and molecular weight distribution (MWD). Recently studies by different investigators ${ }^{1-3}$ have been aimed at elucidating the molecularweight-distribution dependence of the viscoelastic response of high-MW polymer systems. These rheological experiments represent a form of mechanical spectroscopy for the examination of interactions between molecules of different sizes. Synthetic molecular weight distributions have been created by blending different components of defined MW. This technique allows

* Pressent address: Rochester Corporate Research Laboratory, Xerox Corporation, Rochester, New York, N.Y., 14603, U.S.A. the investigator to follow the response of each component in the changing environment created by different compositional ratios.

Two distinct blending regions have now been experimentally defined. ${ }^{1}$ They are characterized by the MW dependence of the steady-state shear compliance, $J_{\mathrm{e}}^{\circ}$, of samples with narrow MWDs, where $J_{\mathrm{e}}^{\circ}$ is defined as the low-frequency limit of the storage compliance, $J^{\prime}$. One region consists of blends of relatively low-MW polymers in which the components have different values of $J_{\mathrm{e}}^{\circ}$. Typically the compliance of these components is proportional to molecular weight, as predicted by the modified Rouse theory for polymer melts. ${ }^{4}$ At high MWs the steady-state shear compliance of systems with similar 
The Effects of High-Molecular-Weight Compouents on the Viscoelastic Properties of Polystyrene

MWDs is relatively independent of $\mathrm{MW}^{1,3,5-7}$. This second region involves strong interactions between molecules, i. e., "entanglements". Studies of blends in tanglement region show that $J_{e}^{\circ}$ is a strong function of MWD. The $J_{\ominus}^{\circ}$ of binary blends passes through a very high maximum at low values of $w_{2}$, the weight fraction of the high-MW component. Beyond this maximum it has been previously shown that $J_{\mathrm{e}}^{\circ}$ is proportional to $w_{2}^{-2}$ or $M_{w}^{-2}$ of the blends. ${ }^{3,1}$ To date, however, only one set of experiments have actually shown the shape of the maximum in $J_{\mathrm{e}}^{\circ}$ as well as the rapid fall off of $J_{\mathrm{e}}^{\circ}$ at lower values of $w_{2}{ }^{1}{ }^{2}$ These earlier experiments were performed with blends of polydisperse poly(dimethylsiloxane) samples, where the MWD of the components obscured the interactions between the blended species. Other prior blending experiments with narrow-MWD components have chiefly delt with the compositional range beyond the maximum in $J_{\mathrm{e}}^{\circ}$.

The purpose of this study has been to examine the low end of the compositional range (i.e., the region of small $w_{2}$ ) in the entanglement region. The goal is to study the interactions responsible for the maximum in $J_{\ominus}^{\circ}$ as a function of MWD. In addition this study seeks to test the various blending laws which have been proposed to explain the viscoelastic response of high-MW polydisperse systems.

\section{EXPERIMENTAL}

The high-MW, narrow-MWD polystyrene samples used to create the synthetic MWDs in this study were obtained from the Pressure Chemical Company, Pittsburgh, Pa. Previous

Table I. Molecular weight distribution of the blends

\begin{tabular}{crcc}
\hline Sample & $M_{w} / 10^{3}$ & $M_{w} / M_{n}$ & $M_{z} / M_{w}$ \\
\hline 97.2 & 97.2 & 1.04 & 1.04 \\
$0.023 / 411 / 97.2$ & 104.4 & 1.10 & 1.25 \\
$0.033 / 411 / 97.2$ & 107.6 & 1.12 & 1.32 \\
$0.049 / 411 / 97.2$ & 112.5 & 1.16 & 1.42 \\
$0.089 / 411 / 97.2$ & 125.1 & 1.25 & 1.57 \\
$0.209 / 411 / 97.2$ & 162.7 & 1.46 & 1.68 \\
$0.399 / 411 / 97.2$ & 222.3 & 1.66 & 1.54 \\
411 & 411 & 1.04 & 1.05 \\
\hline
\end{tabular}

Polymer J., Vol. 4, No. 2, 1973 studies have shown that the steady state shear compliance of polystyrene samples with narrow MWDs is approximately independent of MW above a molecular weight of about $1000000^{3-7}$ The blended components were chosen to be in this range. The MW characteristics of the components are listed in Table I along with the MW averages of the blends, calculated by the Peticolas and Menefee technique. ${ }^{8}$ The identification numbers of the blends represent the weight fraction of the high-MW component/the MW in thousands of the high-MW component/ and the MW in thousands of the low-MW component. Samples were prepared by dissolving weighed amounts of the components in benzene at a concentration of $1-2 \%$ by volume. The solutions were gently agitated for $24 \mathrm{hr}$ and then freeze-dryed. This technique was chosen to avoid possible sample fractionation which might result from extraction methods such as solvent evaporation or nonsolvent precipitation. The resultant blends and components were compacted into disks and molded at $170^{\circ} \mathrm{C}$ at 10000 psi for two minutes.

The viscoelastic properties of the samples were measured with a model R-17 Weissenberg Rheogoniometer used in the cone and plate configuration. A $4^{\circ}$ cone, $2.5 \mathrm{~cm}$ in diameter was chosen to minimize sample size and to optimize the normal stress measurements. The dynamic properties of the samples were measured with an improved version of the Birnboim Ultra Low Frequency Phase Meter ${ }^{9}$. The modifications incorporate variable trigger levels which allow the operator to examine different portions of the input signals. In this manner the purity and balance of the wave forms may be checked and any perturbation or distortion in the signals may be detected and avoided. The modified phase meter defined phase angles to less than \pm 0.2 degrees and determined the amplitudes of the sinosoidal signals to within $\pm 2 \%$.

Different strain amplitudes were used to insure that all measurements were conducted in the region of linear viscoelastic response. Generally measurements were made at maximum strains of $5-9 \%$. 


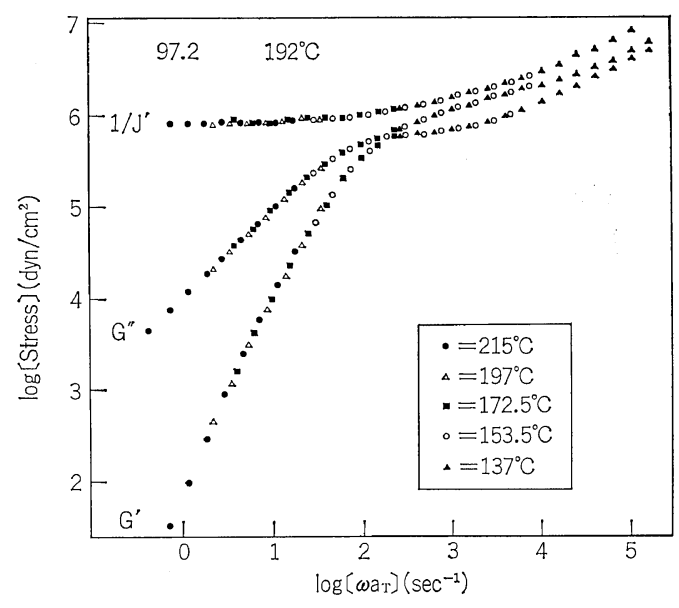

Figure 1. Frequency dependence of $G^{\prime}, G^{\prime \prime}$, and $1 / J^{\prime}$ for sample 97.2 reduced to $192^{\circ} \mathrm{C}$.

\section{RESULTS}

\section{Viscoelastic Response}

Figure 1 shows the viscoelastic response of the narrow MWD polystyrene component with a MW of 97200. All data was reduced to a reference temperature, $T_{0}=192^{\circ} \mathrm{C}$, by multiplying the measured stresses by $\rho_{0} T_{0} / \rho T$. The densities, $\rho$, were calculated from the relationship given by Fox and Loshack. ${ }^{10}$ The reference temperature was chosen to correspond to a convenient value of the zero shear viscocity.

The master curve was constructed from measurements made at five different temperatures. The reduced data was superimposed by multiplying the frequency axis by a factor $a_{\mathrm{T}}$. At low frequencies the loss modulus, $G^{\prime \prime}$, is proportional to $\omega$ and the storage modulus, $G^{\prime}$ is proportional to $\omega^{2}$. At the reference temperature of $192^{\circ} \mathrm{C}$, the low-frequency limit of the dynamic viscosity $\eta^{\prime}=G^{\prime \prime} / \omega$ is $10^{4}$ poise and the

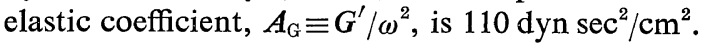
The storage compliance $J^{\prime}$, can be calculated from $G^{\prime}$ and $G^{\prime \prime}$ by eq 1 .

$$
J^{\prime}=\frac{G^{\prime}}{G^{\prime 2}+G^{\prime 2}}
$$

The reciprocal of $J^{\prime}$ is shown in Figure 1 to be a constant, with a value of $9 \times 10^{5} \mathrm{dyn} / \mathrm{cm}^{2}$, over the two lowest decades of frequency. This value is the reciprocal of $J_{\mathrm{e}}^{\circ}$.

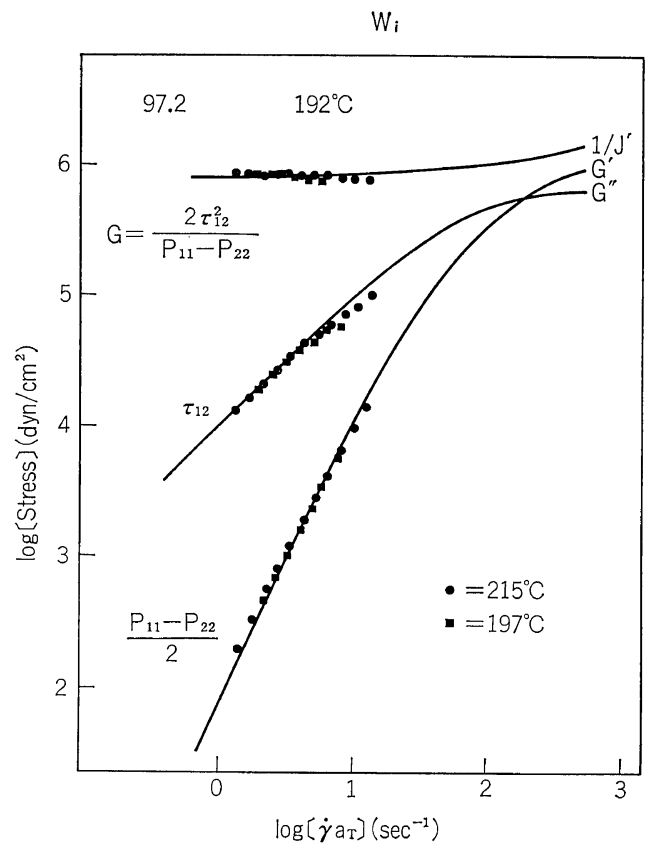

Figure 2. Shear rate dependence of $\tau_{12},\left(\boldsymbol{P}_{11}-\boldsymbol{P}_{22}\right) /$ 2 , and $G$ compared with the frequency dependence of $G^{\prime}, G^{\prime \prime}$, and $1 / J^{\prime}$ for sample 97.2 reduced to $192^{\circ} \mathrm{C}$.

The measurements of the storage modulus extend down to $32 \mathrm{dyn} / \mathrm{cm}^{2}$ which, in this instance, corresponds to a phase angle of 89.7 degrees between the stress and the strain. The lack of scatter in $1 / J^{\prime}$ at low frequencies is an indication of the precision of the measurements of the phase angle, $\phi$. In this region $1 / J^{\prime}$ is proportional to the secant of $\phi$ which approaches infinity as $\phi$ approaches 90 degrees. A tenth of a degree error in the measurement of $\phi$ at 89.7 degrees would change $1 / J^{\prime}$ by $33 \%$, a variation which would be very noticeable in Figure 1.

The data points in Figure 2 give the steadystate shear measurements of the shear stress $\tau_{12}$, half the primary normal stress difference $\left(\boldsymbol{P}_{11}-\right.$ $\left.\boldsymbol{P}_{22}\right) / 2$, and the shear modulus $G=2 \tau_{12} /\left(\boldsymbol{P}_{11}-\boldsymbol{P}_{22}\right)$ as a function of the reduced shear rate $\dot{\gamma} a_{\mathrm{T}}$. These are compared with lines which represent the dynamic measurements of $G^{\prime}, G^{\prime \prime}$, and $1 / J^{\prime}$ as a function of $\omega a_{\mathrm{T}}$, (see Figure 1). The superposition of the steady-state shear data by the shift factor $\boldsymbol{a}_{\mathrm{T}}$, indicates that $\tau_{12}$, and $\boldsymbol{P}_{11}-\boldsymbol{P}_{22}$ have the same temperature dependence as the 
The Effects of High-Molecular-Weight Components on the Viscoelastic Properties of Polystyrene

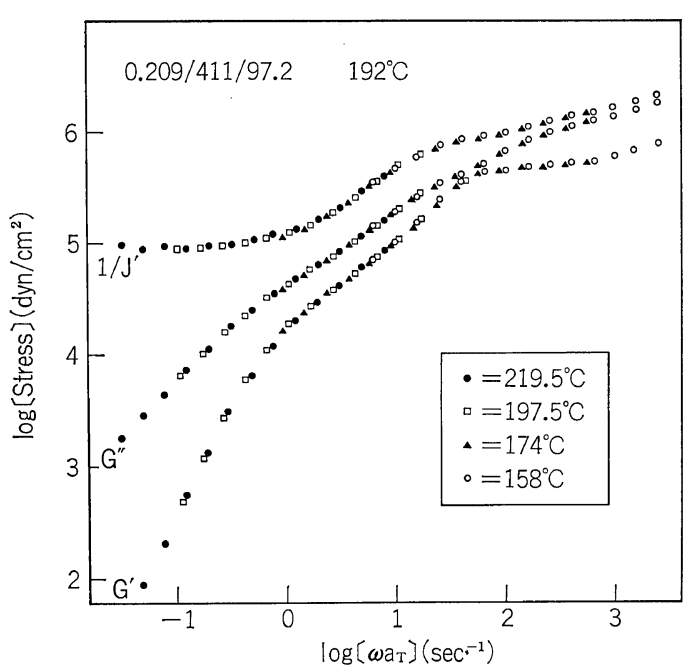

Figure 3. Frequency dependence of $G^{\prime}, G^{\prime \prime}$, and $1 / J^{\prime}$ for sample $0.209 / 411 / 97.2$ reduced to $192^{\circ} \mathrm{C}$.

viscoelastic properties. The data shows that for this system $G^{\prime}$ equals half the primary normal stress difference. At low shear rates $\tau_{12}$ is equal to $G^{\prime \prime}$. At higher shear stresses the samples extrude from the test region making the calculated $\tau_{12}$ appear to fall below $G^{\prime \prime}$. This figure shows that the shear modulus determined in steady state flow $G$, is equal to the low-frequency limit of $1 / J^{\prime}$, in accordance with the predictions of the Theory of Second Order Fluids. ${ }^{11}$

Figure 3 presents the viscoelastic response of a blend of two narrow MWD samples which was formed by adding a high-MW component to the 97200 sample described above. The identification number incates that the blend consists of 20.9 weight percent of a sample with a molecular weight of 411000 mixed with the 97200-MW component. At low frequencies $G^{\prime}$ and $G^{\prime \prime}$ are shown to be proportional to $\omega^{2}$ and $\omega$ respectively, as was found for the base $97200-$ MW sample. The time dependence of this response is shifted to lower frequencies relative to the resfonse of the base component (see Figure 1), reflecting the increased viscosity and the changes in the elastic properties caused by blending. The zero shear viscosity $\eta_{0}$, of this blend is $5.43 \times 10^{4}$ poise and the elastic coefficient is $3.1 \times 10^{4} \mathrm{dyn} \mathrm{sec} / \mathrm{cm}^{2}$ at $192^{\circ} \mathrm{C}$. At intermediate frequencies the effects of blending be- come apparent when both $G^{\prime}$ and $G^{\prime \prime}$ deviate from the low $\omega$ dependencies. At this point both the position and the shape of the frequency response differs from that seen in Figure 1 for the low MW component. At high frequencies all functions have the same shaped response as the properties of the $97200-\mathrm{MW}$ sample. The entire response can be viewed as a weighted combination of the viscoelastic properties of each component. At low frequencies the 411000MW component controls the magnitude of the viscous and elastic response. At high frequencies the properties of the blend are those of the 97200-MW, shifted towards longer times by the interactions with the high MW component. Figure 3 shows that as $\omega$ decreases, $1 / J^{\prime}$ first approaches the limiting value of $9 \times 10^{5} \mathrm{dyn} / \mathrm{cm}^{2}$ which is the value of $1 / J_{\mathrm{e}}^{\circ}$ found for the 97200 MW sample. The effects of blending then appear as a sudden decrease in the magnitude of $1 / J^{\prime}$ with decreasing $\omega$, rultimately reaching a limiting value of $9.5 \times 10^{4} \mathrm{dyn} / \mathrm{cm}^{2}$, the characteristic $1 / J_{\mathrm{e}}^{\circ}$ of the blend. For this blending ratio the compliance changes by almost a decade with a $20.9 \%$ change in composition while the viscosity increases by a factor of 5.43 .

The steady-state shear response of the 0.209 / 411/97.2 blend is compared, in Figure 4, with lines which represent the dynamic properties $G^{\prime}$,

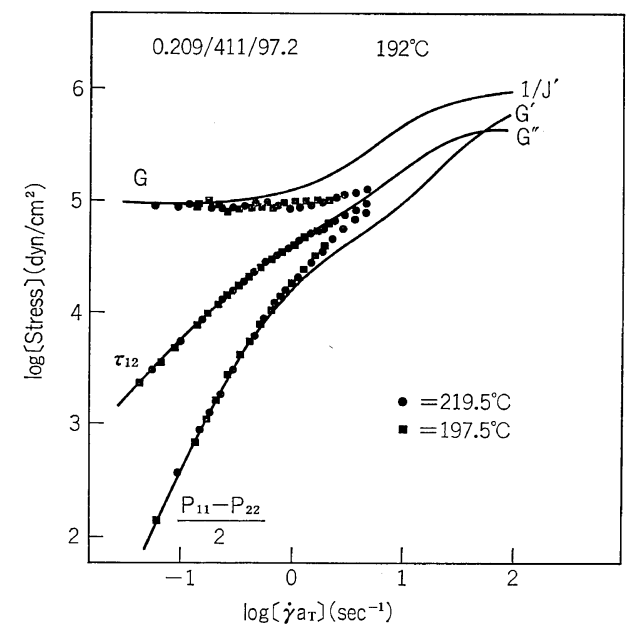

Figure 4. Shear rate dependence of $\tau_{12},\left(\boldsymbol{P}_{11}-\boldsymbol{P}_{22}\right) / 2$ and $G$ compared with the frequency dependence of $G^{\prime}, G^{\prime \prime}$, and $1 / J^{\prime}$ for sample 0.209/411/97.2-reduced to $192^{\circ} \mathrm{C}$. 


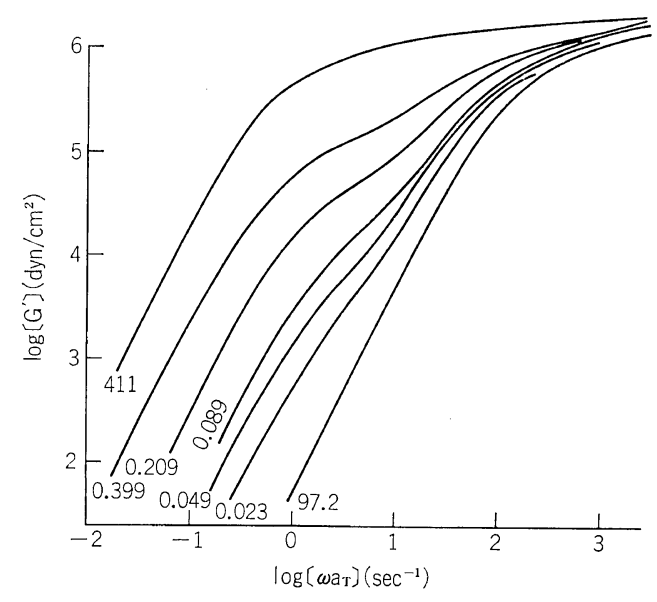

Figure 5. Frequency dependence of $G^{\prime}$ for the $411 / 97.2$-blend series reduced to $192^{\circ} \mathrm{C}$.

$G^{\prime \prime}$, and $1 / J^{\prime}$. At low shear rates $\left(P_{11}-P_{22}\right) / 2$ equals $G^{\prime}$ and is proportional to $\dot{r}^{2}$. At higher shear rates $G^{\prime}$ deviates from the square dependence sooner and more rapidly than $\boldsymbol{P}_{11}-\boldsymbol{P}_{22}$. This reflects the differences between the linear response of the dynamic measurements and the nonlinear response of the steady-state shear experiment. The modulus calculated from the primary normal stress difference is independent of $\dot{\gamma}$ over most of the experimentally accessible range. At low $\omega, G$ equals $1 / J_{\mathrm{e}}^{\circ}$ again in agreement with the Theory of Second Order Fluids. This fact was confirmed for all of the blending experiments described in this paper. Note that $1 / J^{\prime}$ departs from the limiting value of $1 / J_{e}^{\circ}$ at lower shar stresses than does $G$. Thus a good estimate of $1 / J_{\mathrm{e}}^{\circ}$ can even be made from meas-

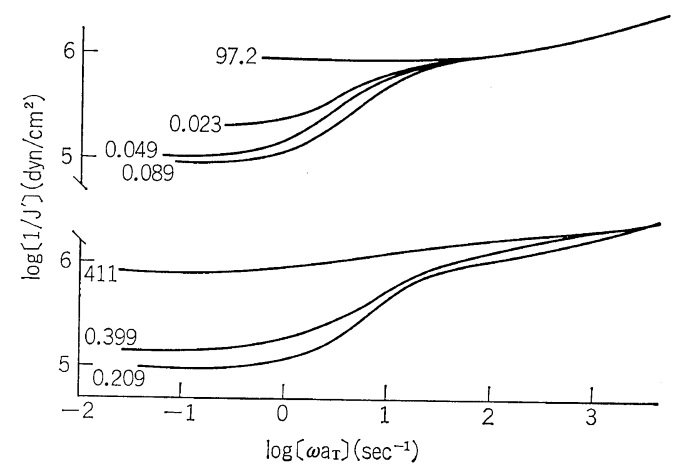

Figure 6. Frequency dependence of $1 / J^{\prime}$ for the $411 / 97.2$-blend series reduced to $192^{\circ} \mathrm{C}$. urements of a constant value of $G$ in the nonlinear region of $\tau_{12}$.

The changes in the response of $G^{\prime}$, caused by the blending of different molecular weight species, are shown in Figure 5. As more of the 411000 -MW component is added to the 97200$M W$ base sample, the response of the system shifts to lower frequencies and an inflection point appears in $G^{\prime}$. The magnitude of the stress at the inflection point increases with $w_{2}$ and the initial formation of a plateau becomes apparent. In this region the response of the blend can be visually decomposed into the contributions of each component.

The blending induced changes in $1 / J^{\prime}$ are given by the family of curves presented in Figure 6. Two distinct concentration regions are observed. For blends with small amounts of high-MW material, the low $\omega$ limit of $1 / J^{\prime}$ decreases rapidly with increasing $w_{2}$, while the high frequency response remains essentially unchanged. The location of the transition between the two extremes shifts to higher frequencies as $w_{2}$ increases. At higher concentrations the magnitude of both the low-and high-frequency responses increase with $w_{2}$ and the position of the transition region shifts toward lower $\omega$.

The shear rate dependencies of $\boldsymbol{P}_{11}-\boldsymbol{P}_{22}$ and $G$ for the $411 / 97.2$ blend series are shown in Figure 7. As the concentration of the high-

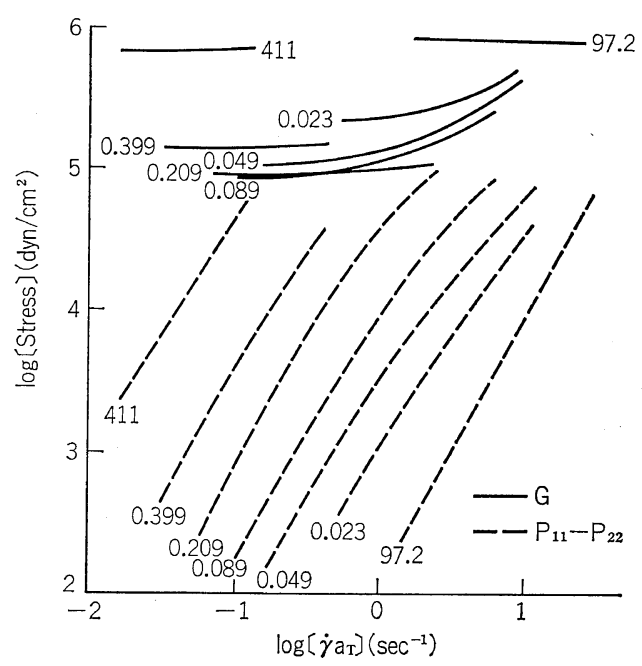

Figure 7. Shear rate dependence of $G$ and $P_{11}-$ $P_{22}$ for the $411 / 97.2$-blend series reduced to $192^{\circ} \mathrm{C}$. 
MW material is decreased the deviation of $P_{11}-$ $\boldsymbol{P}_{22}$ from the $\dot{\gamma}^{2}$ dependence occurs at progressively lower stress levels. However, these deviations are minor when compared with the associated rapid change in $G^{\prime}$ (see Figure 4 and 5). In addition, the shear-rate dependence of $\boldsymbol{P}_{11}-\boldsymbol{P}_{22}$ does not indicate the presence of the two MW species as clearly as does $G^{\prime}$. Therefore the high-shear-rate dependence of the primary normal stress difference is controlled primarily by the high-MW component.

For the narrow MWD components, the shear modulus is independent of $\dot{\gamma}$ over the experimentally accessible range. The measured $G$ remains relatively insensitive to $\dot{\gamma}$ for the blends in which $w_{2}>0.2$. The shear moduli of blends containing small amounts of high-MW material is constant at low $\dot{\gamma}$ but increases rapidly at higher shear rates. This curvature is the result of the more rapid deviation of $\boldsymbol{P}_{11}-\boldsymbol{P}_{22}$ from $\dot{\gamma}^{2}$ than $\tau_{12}$ from $\dot{\gamma}$. For large concentrations of the high-MW component, the deviation of $P_{11}$ $\boldsymbol{P}_{22}$ is balanced by pronounced non-Newtonian

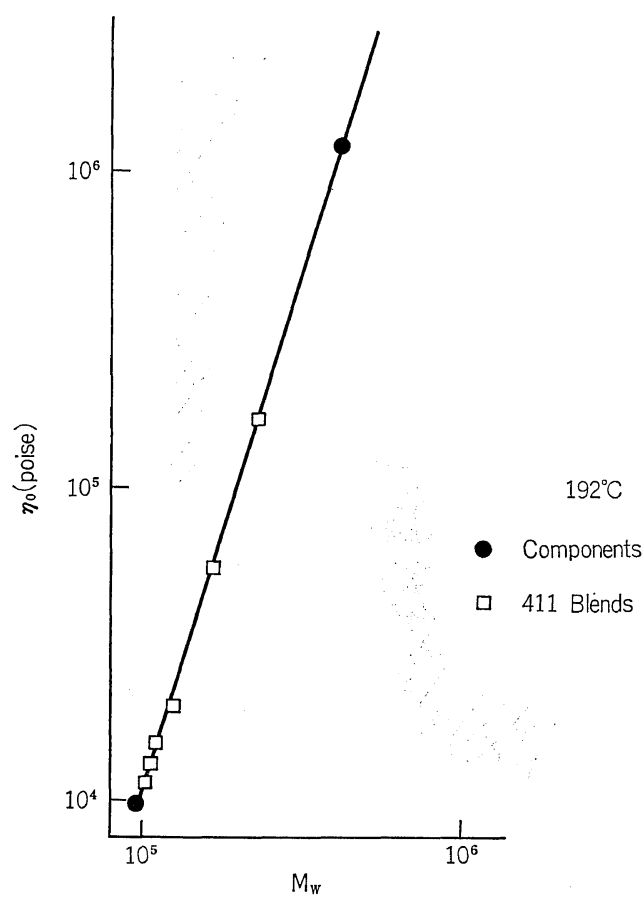

Figure 8. The weight-average molecular weight dependence of the zero shear viscosity for the 411/97.2-blend series. flow (see Figure 4), resulting in a relatively constant value of $G$. The less pronounced nonNewtonian flow in the low $w_{2}$ blends makes $G$ more sensitive to the shear rate dependence of $\boldsymbol{P}_{11}-\boldsymbol{P}_{22}$. Presumably, if higher normal stresses could be measured, the shear modulus of the low $w_{2}$ blends would become less dependent on $\dot{\gamma}$ in the region of substantial non-Newtonian flow. As in Figure 4, the shear moduli of all the blends are less dependent on $\dot{\gamma}$ than $J^{\prime}$ is on $\omega$. This reflects difference between the time dependencies of $P_{11}-P_{22}$ and $G^{\prime}$ and reinforces the previously stated observation that $1 / J_{\mathrm{e}}^{\circ}$ can be estimated from the nonlinear region of steady state flow.

\section{Zero Shear Viscosity}

This study provides an important test of the MW averages which control the long time properties of high-MW polymer melts because it deals primarily with the low $w_{2}$ range, the region in which the ratios of the higher $\mathrm{MW}$ averages, $M_{z} / M_{w}, M_{z+1} / M_{z}$, reach a maximum. Berry and Fox ${ }^{12}$ have shown that the zero shear viscosity $\eta_{0}$, is a unique function of the chain length of the molecule when the systems are reduced to a constant free volume. This con-

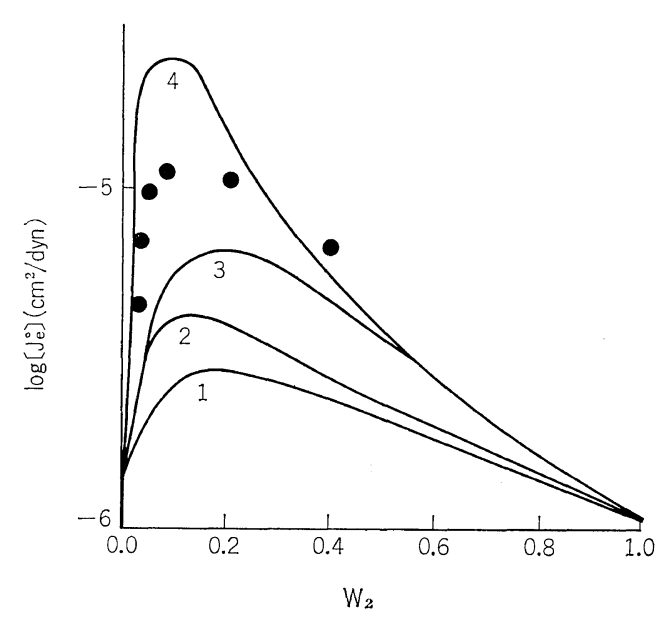

Figure 9. The dependence of the steady-state shear compliance on the weight fraction of the high-MW component of the 411/97.2-blend series. Solid lines are the predictions of (1) Bogue, et al., quadradic blending law; (2) Modified Rouse theory; (3) Mills' correlation; (4) Graessley's theory. 
dition is automatically satisfied in this study by the high MWs of the blended components. For all of the samples studied, the steady state shear measurement of $\eta_{0}$ equaled the low frequency limit of $\eta^{\prime}$. Figure 8 presents the zero shear viscosities of the blends and the components at $192^{\circ} \mathrm{C}$, plotted against the weight-average molecular weight $M_{w}$. The solid line, determined by the viscosities of the narrow MWD samples, is given by the relationship

$$
\log \eta_{0}=-12.7+3.35 \log M_{w}
$$

Within experimental error, the viscosities of all the blends obey this relationship. The least squares line determined by all the data is:

$$
\log \eta_{0}=-13.0+3.41 \log M_{w}
$$

These equations are in agreement with the findings of a recent survey $^{13}$ which compared several investigators' measurements of the viscosity of these and other standard polystyrene samples. Bueche ${ }^{14}$ has predicted that the MW averages which control $\eta_{0}$ shift from $M_{w}$ to $M_{z}$ as the higher moments of the blends would show a systematic deviation from the $M_{w}{ }^{3,4}$ dependence as the ratio of $M_{z} / M_{w}$ passes through a maximum. This is not observed experimentally.

\section{Steady-State Shear Compliance}

Several different predictions for the MWD dependence of $J_{\mathrm{e}}^{\circ}$ have been proposed..$^{3,16,20,22}$ Two of these define blending laws which describe the viscoelastic response of the blend in terms of $H(\tau)$, the relaxation spectrum of the system. This quantity is particularly useful because it can be used to calculate the entire response of all the viscoelastic functions. Ninomiya ${ }^{16}$ has proposed a blending law, eq 4 , in which the relaxation spectrum of the blend is described as a weighted sum of the relaxation spectra of the individual components, each shifted in time by an amount $\lambda$.

$$
H_{\mathrm{B}}(\tau)=\sum v_{i} H_{i}\left(\tau / \lambda_{i}\right)
$$

These shift factors take into account the different environment that a molecule encounters when immersed in different MW surroundings. In a monodisperse sample, a molecule "sees" certain viscous drag forces which determine the time constants of its relaxation mechanisms. In a blend each molecule experiences a new set of viscous drag forces which establish a new set of relaxation times. The relation spectrum of the molecule is then shifted in time by an amount determined by these new interactions. This shift in time scale can be represented by the parameters $\lambda$.

Ninomiya and Ferry's ${ }^{15-18}$ work primarily involved blends of components with different moduli. For these systems, it was found that the weighting factors, $v_{i}$, were equal to the weight fractions of the components. Coupling this result with the observation that $\eta_{\circ}$ B depends only on $M_{w}$ leads to the prediction that the compliance of the blend $J_{\mathrm{e}}^{\circ}$, is proportional to $\left(M_{z+1} M_{z} / M_{w}\right)$ for systems in which $J_{\mathrm{e} i}^{\circ} \alpha M$. This relationship is generally observed. When $J_{\mathrm{e}_{i}}^{\circ}$ is independent of $M$, the blending law predicts $J_{\mathrm{eB}}^{\circ} \alpha M_{z} / M_{w}$ or in another modification, ${ }^{19}$ $J_{\mathrm{eB}}^{\circ} \alpha\left(M_{z+1} M_{z}\right) / M_{w}{ }^{2}$. Previous studies by these ${ }^{1}$ and other authors ${ }^{2,3}$ have shown that these MW averages grossly underestimate the magnitude of the changes in $J_{\mathrm{eB}}^{\circ}$ and the dependence of $J_{\mathrm{e} 3}^{\circ}$ on $w_{2}$. Experimentally $J_{\mathrm{eB}}^{\circ}$ is found to be approximately proportional to $w_{2}^{-2}$ for large values of $w_{2}{ }^{1,12}$ In addition Masuda, et al., ${ }^{2}$ found a spread in the relaxation spectrum of the blends. They postulated that this spread was the result of contributions to $H_{\mathrm{B}}(\tau)$ from relaxation mechanisms involving couplings between the high- and low-MW components.

Based on these observations, Bogue and coworkers ${ }^{20}$ suggested the quadratic blending law given in eq 5 .

$$
H_{\mathrm{B}}=w_{1}^{2} H_{11}+2 w_{1} w_{2} H_{12}+w_{2}^{2} H_{22}
$$

where $H_{i j}$ is the relaxation spectrum caused by interactions between molecules of MW $i$ and $j$. The shift factors $\lambda_{i j}$, do not appear in this formalism, but are indirectly incorporated in terms of a predefined MW dependence of the relaxation times, $\tau_{i j} \alpha M_{i} M_{j} M_{w}^{1.5}$. This approach predicts that

$$
J_{\mathrm{eB}}^{\circ}=\left(M_{z} / M_{w}\right)^{2} J_{\mathrm{e} i}^{\circ}
$$

In work unrelated to the specific form of the blending laws, Mills ${ }^{3.21}$ has found a correlation between $J_{\mathrm{eB}}^{\circ}$ and $\left(M_{z} / M_{w}\right)^{3.7}$.

A theoretical attempt to explain the MW and 
The Effects of High-Molecular-Weight Components on the Viscoelastic Properties of Polystyrene

MWD dependence of $J_{\mathrm{eB}}^{\circ}$ has recently been proposed by Graessley. The theory introduces intermolecular interactions into the Rouse model by considering both the elastic contributions of entanglements and the viscous drag forces at these points. The drag forces depend on the lengths of the interacting chains. This results in a non-uniform drag coefficient for polydisperse systems. The sum of the relaxation times caused by these interactions is in the form of the quadratic blending law. Averages of $\tau$ over each type of relaxation process automatically include the shift factors.

Figure 9 compares the measured compliances of the 411/97.2 series of blends with the predictions described above. The lines represent the results of: (1) The quadradic blending law, $\left(M_{z} / M_{w}\right)^{2} ;$ (2) The modified Rouse theory, $\left(M_{z+1} M_{z} / M_{x}^{2}\right)$; (3) Mills' correlation, $\left(M_{z} / M_{w}\right)^{3.7}$; and (4) Graessley's theory.

The quadratic blending law predicts the least pronounced dependence of $J_{\mathrm{eB}}^{\circ}$ on MWD. This is particularly surprising because this law was designed to explain the experimentally observed dependence of $J_{\mathrm{EB}}^{\circ}$ on $w_{2}{ }^{-2}$. However larger compliances may be predicted by the quadratic blending law if the shift factors, i.e., the relaxation times of the individual molecular species, are more sensitive to the MWD of the melt than the proposed $M_{i} M_{j} M_{w}{ }^{1.5}$ dependence. This is illustrated by the predictions of Graessley's theory which incorporates calculated $\lambda_{i j}$ with a form of the quadradic blending low. While Graessley's theory overestimates the maximum change in $J_{\mathrm{eB}}^{\circ}$, it is the only representation which approximates the shape and the magnitude of the observed response on either side of

Table II. Properties of the blend at $192^{\circ} \mathrm{C}$

\begin{tabular}{cccc}
\hline$w_{2}$ & $\begin{array}{c}\eta_{\circ \mathrm{B}} / 10^{4}, \\
\text { poise }\end{array}$ & $\begin{array}{c}J_{\mathrm{eB}}^{\circ} / 10^{-6}, \\
\mathrm{~cm}^{2} / \mathrm{dyn}\end{array}$ & $\begin{array}{c}\eta^{*} \eta_{\circ} \mathrm{B} \\
\left(\text { at } \eta_{\circ \mathrm{B}} J_{\mathrm{eB}}^{\circ}=1\right)\end{array}$ \\
\hline 0.000 & 1.00 & 1.1 & 0.67 \\
0.023 & 1.15 & 4.5 & 0.85 \\
0.033 & 1.27 & 7.0 & 0.85 \\
0.049 & 1.51 & 9.0 & 0.83 \\
0.089 & 1.97 & 11. & 0.76 \\
0.209 & 5.43 & 10.5 & 0.67 \\
0.399 & 16.0 & 6.7 & 0.66 \\
1.000 & 125. & 1.4 & 0.68 \\
\hline
\end{tabular}

the maximum.

\section{Non-Newtonian Response}

The authors have previously reported that the non-Newtonian response of many polymer melts is related to the characteristic relaxation time of the system, $\tau=\eta_{0} J_{\mathrm{e}}^{\circ 23}$. A correlation was found between $\tau$ and the reduced complex viscosity, $\eta^{*} / \eta_{0}=\left(G^{\prime 2}+G^{\prime 2}\right)^{1 / 2} / \omega \eta_{0}$. In particular, at $\omega=1 / \tau, \eta^{*} / \eta_{0}=0.67 \pm 0.03$. It was noted that this relationship did not apply to systems containing small amounts of high MW material. The data presented in this paper can be used to examine this behavior in more detail. The reduced complex viscosities of the PS blends are plotted as a function of $\omega a_{\mathrm{T}}$ in Figure 10. The point ${ }^{(0)}$ on each response corresponds to $w a_{\mathrm{T}}=1 / \eta_{0} J_{\mathrm{e}}^{\circ}$. The values of $\eta^{*} / \eta_{\circ}$ at this point are listed in Table II.

As the 97200-MW Polystyrene is added to the higher-MW sample, the Newtonian response extends to progressively higher values of $\omega a_{\mathrm{T}}$. In addition, the presence of the low-MW material initially changes the shape of the nonNewtonian response at low values of $\eta^{*} / \eta_{0}$. This contribution becomes more significant as $w_{2}$ decreases. The shape of the response in the neighborhood of $\eta^{*} / \eta_{0}=0.67$ becomes a function of the low-MW component when $w_{2}<0.2$. As shown in Figure 9, this is the concentration range where $J_{\mathrm{eB}}^{\circ}$ is a maximum, and thus the region in which the elastic properties of the 97200-MW sample first begin to influence the properties of the blend. At small $w_{2}$ the correlation fails when the properties of the lowMW component dominate the response of the blend. However, in this region $\omega=1 / \eta^{\circ} J_{\mathrm{e}}^{\circ}$ pre-

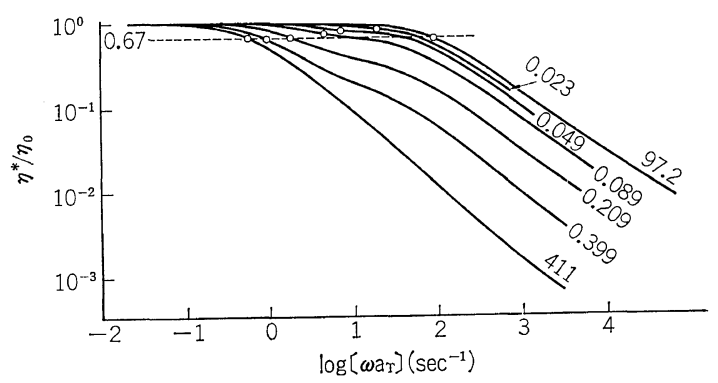

Figure 10. The reduced complex viscosities of the 411/97.2-blend series as a function of $\omega a_{\mathrm{T}}$. The point ${ }^{\circ}$ represents $\omega a_{\mathrm{T}}=1 / \eta_{\circ} J_{\mathrm{e}}^{\circ}$. 
dicts the approximate position where the contribution of the high-MW component to $\eta^{*} / \eta$ 。 equals 0.67 . This may be seen by shifting the response of the high-MW component so that it corresponds with the initial non-Newtonian flow region of the low $w_{2}$ blends. Therefore the characteristic time, $\eta_{\circ} J_{\mathrm{e}}^{\circ}$, can be considered to be a measure of the time scale of the relaxation processes of the high-MW component.

\section{REFERENCES}

1. W.M. Prest, Jr., J. Polym. Sci., Part A-2, 8, 1897 (1970).

2. T. Masuda, K. Kitagawa, T. Inoue, and S. Onogi, Macromolecules, 3, 116 (1970).

3. N.J. Mills and A. Nevin, J. Polym. Sci., Part $A-2$, 9, 267 (1971).

4. J.D. Ferry, M.L. Williams, and D.M. Sterns, J. Phys. Chem., 58, 987 (1954).

5. J.M. O'Reilly and W.M. Prest, Jr., Trans. Soc. Rheol., 10: 2 (1967).

6. S. Onogi, T. Masuda, and K. Kitagawa, Macromolecules, 3, 109 (1970).

7. H.J.M.H. Mieras and C.F.H. van Rijn, $\mathrm{Na}$ ture, 218, 865 (1968).

8. W.L. Peticolas and E. Menefee, J. Chem. Phys.,
35, 957 (1961).

9. M.H. Birnboim, U. S. Patent 3286176 (Nov. 15, 1966).

10. T.G Fox and S. Loshaek, J. Polym. Sci., 15, 371 (1955).

11. B.D.Coleman and H.Markovitz, J. Appl. Phys., 35, 1 (1964).

12. G.C. Berry and T. G Fox, Advan. Polym. Sci., 5, 261 (1968).

13. A. Casale, R. S. Porter, and J. F. Johnson, $J$. Macromol. Sci.-Revs. Macromol. Chem., C5(2), 387 (1971).

14. F. Bueche, J. Polym. Sci., 43, 527 (1960).

15. K. Ninomiya, J. Colloid Sci., 14, 49 (1959).

16. K. Ninomiya, ibid., 17, 759 (1967).

17. K. Ninomiya and J. D. Ferry, ibid., 18, 421 (1963).

18. K. Ninomiya, J.D. Ferry, and Y. Ôyanagi, $J$. Phys. Chem., 67, 2297 (1963).

19. W.W. Graessley and L. Segal, Macromolecules, 2, 49 (1969).

20. D. C. Bogue, T. Masuda, Y. Einaga, and S. Onogi, Polymer J., 1, 563 (1970).

21. N.J. Mills, Eur. Polym. J., 5, 675 (1969).

22. W.W. Graessley, J. Chem. Phys. 545143 (1971).

23. W.M. Prest, Jr., R.S. Porter and J.M. O'Reil1y, J. Appl. Polym. Sci., 14, 2697 (1970). 